\title{
Magnetic Field Sensor Based on Magnetic Tunnel Junction with Voltage-Tunable Magnetic Anisotropy
}

\author{
W. Skowroński ${ }^{a, *,}$, P. Wiśniowski ${ }^{a}$, J. Wrona ${ }^{a, b}$, A. ŻYWCZAK ${ }^{c}$ AND T. Stobiecki ${ }^{a}$ \\ ${ }^{a}$ AGH University of Science and Technology, Department of Electronics, al. Mickiewicza 30, 30-059 Kraków, Poland \\ ${ }^{b}$ Singulus Technologies, Kahl am Main, 63796, Germany \\ ${ }^{c}$ AGH University of Science and Technology, Academic Center for Materials and Nanotechnology, al. Mickiewicza
} 30, 30-059 Kraków, Poland

\begin{abstract}
We present a submicron magnetic field sensor with voltage-tunable magnetic field sensitivity. The device, based on magnetic tunnel junction, exhibits high tunnelling magnetoresistance ratio of up to $90 \%$. Perpendicular magnetic anisotropy of thin ferromagnetic sensing layer in combination with an in-plane magnetized reference layer is used to obtain linear change in the sensor resistance in response to the in-plane magnetic field. The perpendicular anisotropy is further controlled by the bias voltage and, thus, the sensitivity of the sensor is changed. In addition, we evaluate the sensor selectivity for the magnetic field direction and present an influence of the temperature on the anisotropy.
\end{abstract}

DOI: $10.12693 /$ APhysPolA.127.496

PACS: 85.75.-d, 85.75.Ss, 85.35.-p

\section{Introduction}

Measurement of weak magnetic fields produced by micro- and nano-scale size elements is an important issue in many medical and technical applications, such as magnetoencephalography [1], magnetic nanoparticles detection [2], read head of hard disc drives or integrated circuit leakage current detection [3]. various detection methods were proposed to meet these expectations, such as, Halleffect sensors, superconducting quantum interference devices (SQUID) and magnetoresistance elements. In the last case, magnetic tunnel junction (MTJ) comprising two ferromagnets separated by a thin tunnel barrier is a very promising candidate due to a large tunnelling magnetoresistance (TMR) and high detection range at room temperature [4].

In this work, we present experimental data on $\mathrm{CoFeB} / \mathrm{MgO} / \mathrm{CoFeB} \mathrm{MTJ}$ magnetic field sensor. In order to increase spatial resolution of the device, the MTJ with nanometer-scale size was fabricated. Taking advantage of perpendicular interface magnetic anisotropy, we tuned the thicknesses of both layers to achieve orthogonal magnetization alignment, i.e., perpendicular magnetization for the sensing and in-plane magnetization for the reference layer [5]. The $\mathrm{MgO}$ tunnel barrier was thick enough to hamper any spin-transfer-torque related effects [6]. We achieved almost one order of magnitude tunability ratio, both in sensitivity and in the field detection range, which is higher than in our pre-

\footnotetext{
$\dagger^{\dagger}$ corresponding author; e-mail: skowron@agh.edu.pl
}

$\dagger^{\dagger}$ now at National Institute of Advanced Industrial Science and Technology (AIST), Spintronics Research Center, Tsukuba, Ibaraki 305-8568, Japan vious work [7]. Moreover, we present the results of the magnetic anisotropy change measured in the temperature range from 15 to $400 \mathrm{~K}$. In addition, we show data on the sensor selectivity to the magnetic field direction.

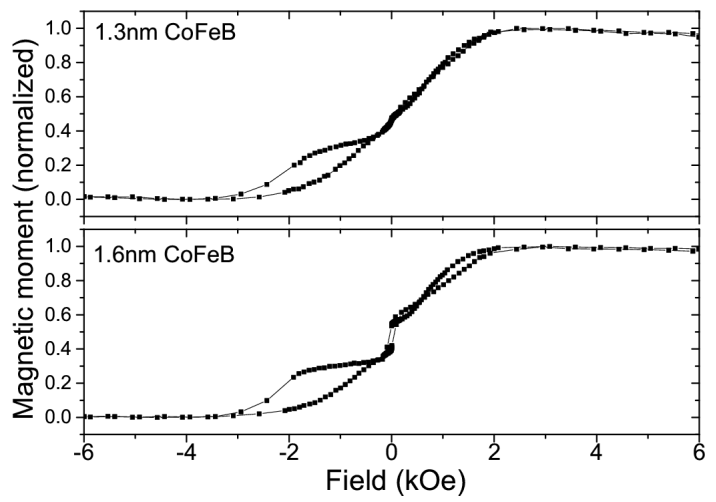

Fig. 1. Magnetization hysteresis loops of unpatterned MTJ multilayers with 1.3 and $1.6 \mathrm{~nm}$ thick FL.

\section{Experiment}

For our studies we used a following multilayer system (from the top): $\mathrm{Ru}(7) / \mathrm{Ta}(10) / \mathrm{Co}_{40} \mathrm{Fe}_{40} \mathrm{~B}_{20}(1.6-$ 1.3) $/ \mathrm{MgO}(1.6) / \mathrm{Co}_{40} \mathrm{Fe}_{40} \mathrm{~B}_{20}(2.3) / \mathrm{Ru}(0.9) / \mathrm{Co}_{70} \mathrm{Fe}_{30}(2.1)$ $/ \mathrm{Pt}_{38} \mathrm{Mn}_{62}(16) / \mathrm{Ta}(3) / \mathrm{CuN}(30) / \mathrm{Ta}(3) / \mathrm{CuN}(30) / \mathrm{Ta}(5)$ $/ \mathrm{SiO}_{2} / \mathrm{Si}$ (substrate), with the thickness denoted in $\mathrm{nm}$, deposited by means of magnetron sputtering using Timaris System from Singulus Technologies [6]. After the deposition, the multilayer system was annealed at $340^{\circ} \mathrm{C}$ in order to induce proper crystallographic orientation of $\mathrm{CoFeB}$ electrodes [8]. An in-plane magnetic field was applied during the annealing and cooling process in order to set and exchange bias direction [9]. Figure 1 presents vibrating sample magnetometer results 
of MTJ with two different thicknesses of the $\mathrm{CoFeB}$ free layer (FL). In-plane hard axis loop is measured for $1.3 \mathrm{~nm}$ thick FL, whereas, in-plane easy axis is observed for $1.6 \mathrm{~nm}$ FL. For the design of our sensor we chose an MTJ with a $1.3 \mathrm{~nm}$ thick FL, as its magnetization orientation is perpendicular to the RL, and thus results in a cross magnetization configuration of the MTJ. The resistance-area product of the MTJ with $1.6 \mathrm{~nm}$ thick $\mathrm{MgO}$ tunnel barrier $\left(t_{\mathrm{MgO}}\right)$ is $2 \mathrm{kOhm} \mu \mathrm{m}^{2}$ and the TMR ratio is up to $90 \%$.

In order to obtain high spatial resolution, the MTJ was patterned into submicrometer size ellipse, with a short and long diameter of 120 and $230 \mathrm{~nm}$, respectively, using electron-beam lithography, ion-beam milling and lift-off process in ISO-5 class cleanroom facilities at Academic Center for Materials and Nanotechnology AGH. Sensing properties were measured in an in-plane magnetic field and the bias voltage $V_{b}$ was applied with respect to the top FL contact. In order to determine the sensor magnetic field orientation selectivity, perpendicular magnetic field was applied.

Magneto-transport properties were also measured at different $V_{b}$ in closed-cycle helium cryostat, in temperatures ranging from 15 to $400 \mathrm{~K}$. The perpendicular magnetic anisotropy energy was calculated using method presented in Ref. [7].

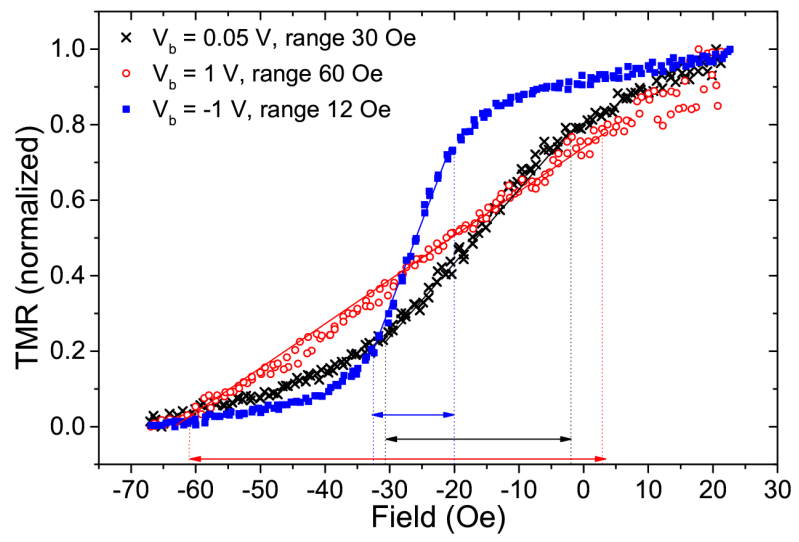

Fig. 2. Normalized TMR ratio vs. in-plane magnetic field measured for different bias voltage. Solid line represents fit to the experimental points within $2 \%$ nonlinearity margin. Lines with arrows indicate linear operation range.

\section{Results and discussion}

Normalized tunnelling magnetoresistance curves, measured for different bias voltage are shown in Fig. 2. For low bias voltage, the linear range, defined as the range within the sensor exhibit linear response to the magnetic field with less than $2 \%$ error, is 30 Oe. A horizontal shift of the resistance vs. magnetic field curve is due to the antiferromagnetic coupling between free and reference layers, originating from the magnetostatic interactions. Sensor linear operation range changes from 12 Oe for $V_{b}=-1 \mathrm{~V}$ up to 60 Oe for $V_{b}=1 \mathrm{~V}$. Likewise, sensor sensitivity, defined as the resistance change in \%, changes from $0.49 \% /$ Oe for $V_{b}=-1 \mathrm{~V}$, down to $0.056 \% /$ Oe for $V_{b}=1 \mathrm{~V}$. Different slope of the resistance vs. magnetic field curve is explained by the perpendicular magnetic anisotropy change of thin $\mathrm{CoFeB}$ FL adjacent to the $\mathrm{MgO}$ insulator [10]. The sign of the anisotropy change is similar to other reports on $\mathrm{Ta} / \mathrm{CoFeB} / \mathrm{MgO}$ structures [11]. We would like to point out, that the opposite sign of the anisotropy change is expected for ferromagnet/insulator bilayers, where the $3 \mathrm{~d}$ ferromagnet is adjacent to the $4 \mathrm{~d}$ metal, such as Ru [12]. Next, we focus on the sensor selectivity for the magnetic field direction within investigated field range. Measurements of the resistance (at negative bias voltage $V_{b}=-1 \mathrm{~V}$ ) vs. in-plane and perpendicular magnetic fields are presented in Fig. 3. In the investigated magnetic field range, the highest change of the resistance measured for perpendicular magnetic field was $0.7 \%$, which is almost two orders of magnitude smaller than the corresponding change for an in-plane magnetic field. Due to a submircon planar size of the sensor, it is possible to detect weak magnetic fields in a specific orientation with a very high lateral resolution.

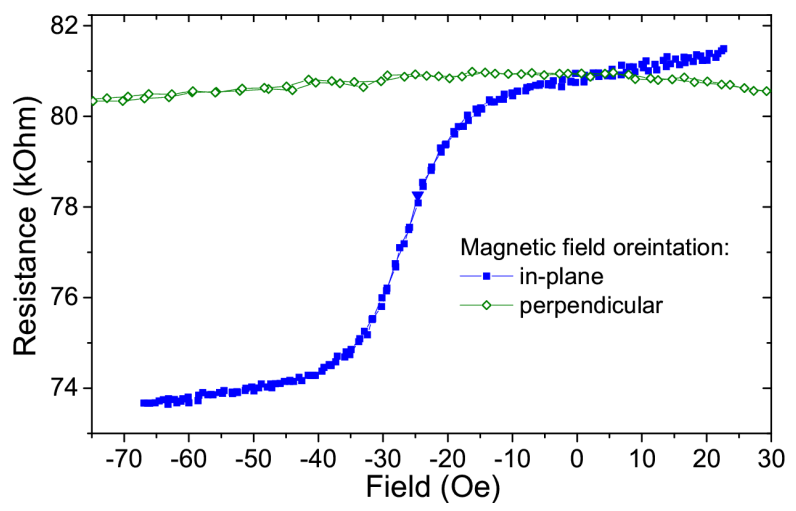

Fig. 3. Resistance vs. magnetic field curves presenting a selectivity to the magnetic field direction of designed MTJ sensor measured for $V_{b}=-1 \mathrm{~V}$.

Finally, the tunalibity of the sensor properties were investigated at temperatures ranging from 13 to $400 \mathrm{~K}$. The perpendicular surface anisotropy calculated from resistance vs. in-plane field measured at different bias voltages and temperatures is presented in Fig. 4. The surface anisotropy energy $K_{i}$ can be calculated using equation:

$$
K_{i}=\left(K_{\mathrm{eff}}-K_{v}\right) t_{\mathrm{FL}}
$$

where, $K_{\text {eff }}$ is the effective anisotropy density derived from the resistance vs. field loop, $K_{v}$ is the volume anisotropy, which is equal to $K_{v}=\left(N_{z}-N_{x}\right) \mu_{0} M_{s}^{2} / 2$, where $N_{z}$ and $N_{x}$ are demagnetization factors, and $t_{\mathrm{FL}}$ is the FL thickness (in this case reduced by the magnetic dead layer thickness of $0.7 \mathrm{~nm}$ ). The antiferromagnetic coupling between free and reference layers was not taken into account in the procedure for determining the surface anisotropy energy, because in our case, the energy term is dominated by the surface and demagnetizing energies. The surface anisotropy energy was 
found to decrease with increasing temperatures, which is consistent with the Bloch law. Likewise, the change of the anisotropy energy with bias voltage, defined as the quotient of the surface anisotropy energy and the electric-field $\left(\Delta E=K_{i} /\left(V / t_{\mathrm{MgO}}\right)\right)$ decreases with increasing temperature, however, more rapidly, which was also observed by Alzate et al. in Ref. [13]. Nevertheless, presented results indicate that the ability to control the sensitivity and field range of the sensor increases with decreasing temperature and is maintained at temperatures up to $400 \mathrm{~K}$.

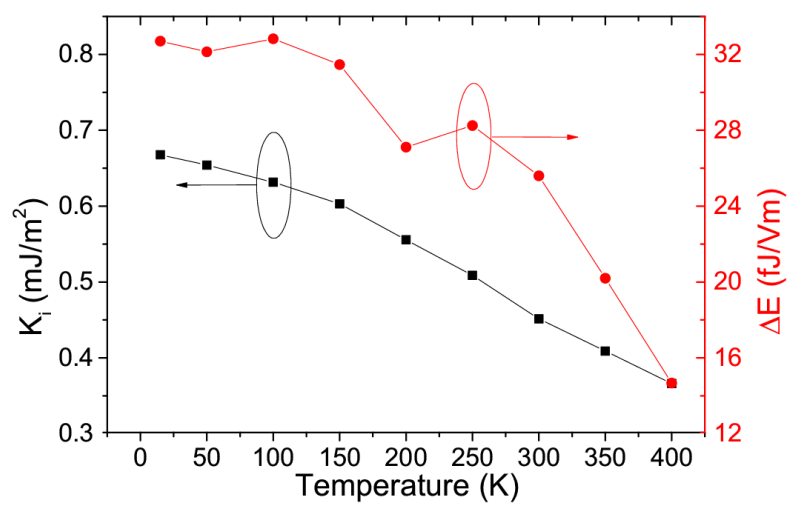

Fig. 4. Temperature dependence of the surface anisotropy energy $\left(K_{i}\right)$ and perpendicular anisotropy energy change per volt $(\Delta E)$.

\section{Conclusions}

In conclusion, we fabricated a nanometric-size magnetic field sensor based on magnetic tunnel junction. We used a perpendicular interface magnetic anisotropy of $\mathrm{Ta} / \mathrm{CoFeB} / \mathrm{MgO}$ free layer structure in order to create an orthogonal magnetization configuration between the sensing and in-plane magnetized reference layers. By varying the bias voltage, we are able to switch between high-sensitivity and low-range to low-sensitivity and high-range sensor operation. We showed that the device is insensitive to perpendicular magnetic field. In addition, we investigate the effect of temperature on magnetic anisotropy energy in the range from 15 up to $400 \mathrm{~K}$.

\section{Acknowledgments}

This work was supported by Polish National Science Center Grant No. DEC-2012/04/M/ST7/00799 and the Statutory Grant No. 15.11.230.140. W.S. acknowledges the Foundation for Polish Science (FNP) scholarship under START Programme.

\section{References}

[1] M. Burghoff, T.H. Sander, A. Schnabel, D. Drung, L. Trahms, G. Curio, B.-M. Mackert, Appl. Phys. Lett. 85, 6278 (2004).

[2] C. Albon, A. Weddemann, A. Auge, K. Rott, A. Hütten, Appl. Phys. Lett. 95, 023101 (2009).

[3] S. Chatraphorn, E.F. Fleet, L.A. Wellstood, F.C. Knauss, T.M. Eiles, Appl. Phys. Lett. $\mathbf{7 6}$, 2304 (2000).

[4] J.M. Almeida, P. Wiśniowski, P.P. Freitas, J. Appl. Phys. 103, 07E922 (2009).

[5] P. Wiśniowski, J.M. Almeida, S. Cardoso, N.P. Barradas, P.P. Freitas, J. Appl. Phys. 103, 07A910 (2008).

[6] W. Skowroński, M. Frankowski, J. Wrona, T. Stobiecki, P. Ogrodnik, J. Barnaś, Appl. Phys. Lett. 105, 072409 (2014).

[7] W. Skowroński, P. Wiśniowski, T. Stobiecki, S. Cardoso, P.P. Freitas, S. van Dijken, Appl. Phys. Lett. 101, 192401 (2012).

[8] D. Djayaprawira, K. Tsunekawa, M. Nagai, H. Maehara, S. Yamagata, N. Watanabe, S. Yuasa, Y. Suzuki, K. Ando, Appl. Phys. Lett. 86, 092502 (2005).

[9] J. Nogues, I.K. Schuller, J. Magn. Magn. Mater. 192, 203 (1999).

[10] T. Maruyama, Y. Shiota, T. Nozaki, K. Ohta, N. Toda, M. Mizuguchi, A.A. Tulapurkar, T. Shinjo, M. Shiraishi, S. Mizukami, Y. Ando, Y. Suzuki, Nat. Nanotechnol. 4, 158 (2009).

[11] Y. Shiota, F. Bonell, S. Miwa, N. Mizuochi, T. Shinjo, Y. Suzuki, Appl. Phys. Lett. 103, 082410 (2013).

[12] S.E. Barnes, J. Ieda, S. Maekawa, Sci. Rep. 4, 4105 (2014).

[13] J.G. Alzate, P.K. Amiri, G. Yu, P. Upadhyaya, J.A. Katine, J. Ocker, B. Langer, I.N. Krivorotov, K.L. Wang, Appl. Phys. Lett. 104, 112410 (2014). 